\title{
Investigation of transmission rate of carbapenemase- producing carbapenem-resistant Enterobacteriaceae among contacted patients and healthcare workers in a tertiary hospital
}

\section{Euijin Chang \\ Ho Eun Chang \\ PHiCS Institute \\ II Seob Shin \\ PHiCS Institute}

Seoul National University Hospital

\section{Young Rok Oh}

Seoul National University Hospital

Chang Kyung Kang

Seoul National University Hospital

Pyoeng Gyun Choe

Seoul National University Hospital

\section{Wan Beom Park}

Seoul National University College of Medicine

\section{Eun Hwa Choi}

Seoul National University College of Medicine

\section{Myoung-don Oh}

Seoul National University College of Medicine

\section{Kyoung Un Park}

Seoul National University College of Medicine

Nam Joong Kim ( $\nabla$ molder@unitel.co.kr)

Seoul National University College of Medicine https://orcid.org/0000-0001-6793-9467

\section{Research}

Keywords: Carbapenemase-producing Enterobacteriaceae, Transmission, Infection control, Whole-genome sequencing

Posted Date: May 6th, 2021

DOI: https://doi.org/10.21203/rs.3.rs-461648/v1

License: (c) (i) This work is licensed under a Creative Commons Attribution 4.0 International License. Read Full License 


\section{Abstract \\ Background}

To reduce transmission of carbapenemase-producing carbapenem-resistant Enterobacteriaceae (CP-CRE), it is recommended that patients and healthcare workers in close contact with CP-CRE-positive patients be screened. The aim of this study was to investigate the transmission rate of CP-CRE to patients and healthcare workers in close contact with CP-CRE-positive patients using whole-genome sequencing (WGS) with remeasurement of the minimum inhibitory concentration (MIC).

\section{Methods}

This study was conducted in a 1,751-bed tertiary teaching hospital between January 2017 and December 2019. The index patients were those found to be CP-CRE-positive during hospitalization and subsequently isolated in single rooms with contact precautions. Screening tests were performed on the patients who shared a room with the index patients for at least one day and the healthcare workers who cared for them. When CRE was isolated, the presence and type of carbapenemase was determined using polymerase chain reaction, and WGS with remeasurement of the MIC was performed to determine the strain type and to verify the association between the CRE and that of the index patient. When the CP-CRE identified in patients or healthcare workers in contact with an index patient had a different type of carbapenemase, the pairs were excluded from genetic analysis.

\section{Results}

A total of 47 index patients were found to have been in contact with 152 patients in shared rooms and 54 healthcare workers. Among the 152 contacted patients, ten had carbapenemase-non-producing CRE and seven had CP-CRE. None of the healthcare workers had CRE (95\% confidence interval 0.0\%-0.1\%). Four patients had the same type of carbapenemase as their index patients, all of which were identified as Klebsiella pneumoniae carbapenemase. WGS with remeasurement of MIC revealed that three of these four patients had genotypes and phenotypes in accordance with the index patient. Consequently, the CP-CRE transmission rate was $2.0 \%$ (95\% confidence interval $0.4-5.6 \%)$.

\section{Conclusions}

The CP-CRE transmission rate between index patients and those in close contact was approximately $2.0 \%$, while there was no transmission of CP-CRE to healthcare workers.

\section{Background}

Since they were developed in 1985, carbapenems have been one of the most widely used antibiotics for several kinds of hospital- and community-acquired infections [1, 2]. With the drastic increase in the number of carbapenems used for treating infections, resistance to carbapenems has been reported more frequently, especially in Enterobacteriaceae [3-5]. Carbapenem-resistant Enterobacteriaceae (CRE) is resistant to at least one of the carbapenems, and is associated with a higher morbidity and mortality than carbapenem-susceptible Enterobacteriaceae [6, 7]. 
In particular, CRE that produce carbapenemases, enzymes that hydrolyze carbapenems, are called carbapenemaseproducing CRE (CP-CRE) [8]. Carbapenem resistance, which is not related to carbapenemase production but is associated with porin deficiency or overexpression of efflux pumps, is practically non-transferable between bacteria $[9,10]$. In contrast, CP-CRE can easily transmit their resistant genes to other bacteria through plasmid transfer [9, 10]. Therefore, CP-CRE is considered a greater hazard to public health than carbapenemase-non-producing CRE (CNP-CRE), and stricter infection control should be implemented when CP-CRE is detected [11].

The Korea Disease Control and Prevention Agency guidelines for controlling the transmission of CP-CRE in hospitals states that if CRE is cultured from a hospitalized patient's specimen, polymerase chain reaction (PCR) tests for detecting carbapenemase genes should also be performed. When carbapenemase genes are detected by PCR, the patient should be isolated in a single room with contact precautions. Moreover, all patients who shared a room overnight and all the healthcare workers who made contact with the patient are required to undergo CRE screening [11].

However, the actual rate of positivity in CP-CRE for patients and healthcare workers in close contact is not well known. A few studies have reported the rate to be between $2.8 \%$ and $3.2 \%[12,13]$. One retrospective study, which reviewed 211 CP-CRE index patients and 1,369 contacted patients between 2010 and 2017 in a tertiary teaching hospital found that carbapenemases were transmitted to 44 contacted patients (3.2\%) [13]. Another prospective study of household contacts between 2015 and 2018 found the rate of CP-CRE transmission to family members to be $2.8 \%$ (5 of 177 contacts) [12].

The aim of this study was to determine the actual rate of positivity in CP-CRE screenings of patients and healthcare workers exposed to CP-CRE-positive patients using whole-genome sequencing (WGS) with remeasurement of the minimum inhibitory concentration (MIC).

\section{Methods}

\section{Study design}

This retrospective study was carried out from January 1, 2017 to December 31, 2019 at the Seoul National University Hospital in the Republic of Korea, a tertiary teaching hospital with 1,751 beds. The MIC of each antibiotic was determined using MicroScan WalkAway (Omron Microscan Systems Inc., Renton, WA, USA). Carbapenem resistance was defined as reduced susceptibility to any carbapenem according to the Clinical and Laboratory Standards Institute criteria (imipenem, meropenem $\geq 2 \mu \mathrm{g} / \mathrm{mL}$ of MIC; ertapenem $\geq 1 \mu \mathrm{g} / \mathrm{mL}$ of MIC) [14]. When CRE was detected in the cultures of any clinical specimens, the Carba NP and PCR tests were conducted to detect carbapenemase genes. The PCR tests targeted carbapenemase genes such as Klebsiella pneumoniae carbapenemase (KPC), imipenemase (IMP), Verona Integron-encoded metallo- $\beta$-lactamase (VIM), New Delhi metallo- $\beta$-lactamase (NDM), and oxacillinase (OXA).

CP-CRE index patients were defined as patients with positive tests for CP-CRE from any infected or colonized site during hospitalization who had contact with other patients in a shared room and healthcare workers before CP-CRE was identified. CP-CRE-contacted patients were those who had shared a room with an index patient for at least one day, and exposed healthcare workers were those that had contact with the patient at least once before they were identified. When CP-CRE was detected, the patient was isolated in a single room with contact precaution regardless of whether CP-CRE was a pathogen or a colonizer. Patients and healthcare workers who were in close contact also underwent CRE screening. The specimens from the contacted patients and healthcare workers were taken as rectal 
swabs. CRE screening tests utilized the disc diffusion method by using meropenem $(10 \mu \mathrm{g})$ discs, with a meropenem-resistance cut-off value of $<25 \mathrm{~mm}$, according to the European Committee on Antimicrobial Susceptibility Testing criteria [15]. When an contacted patient was positive for CRE, a PCR test for carbapenemase genes was performed to check for the presence and type of carbapenemase.

\section{Whole genome sequencing for strain typing}

For strain typing and to verify the genetic association between the CP-CRE-positive index patients and secondary patients (contacted patients who had the same type of carbapenemases as the index patient), WGS was conducted for each CP-CRE index patient-secondary patient pair. Additionally, WGS was performed for each CP-CRE index patient-CNP-CRE detected patient pair to investigate any genetic associations.

Isolates from each CP-CRE index patient-secondary patient pair and CP-CRE index patient-CNP-CRE-detected patient pair were cultured in MacConkey agar plates. Bacterial DNA was extracted using InstaGene Matrix (Bio-Rad Laboratories, Hercules, CA, USA) and the concentration was measured using the QuantFluor ONE dsDNA System (Promega, Madison, WI, USA). The library was prepared using the Nextera DNA Flex kit (Illumina Inc., San Diego, CA, USA) and run on an iSeq ${ }^{\mathrm{TM}} 100$ System (Illumina Inc.) following the manufacturer's instructions. The fastq data obtained were assembled using the Microbial Genomics Module of the CLC Genomics Workbench (Qiagen, Aarhus, Denmark). The same program was then used to align and compare the assembled readings for each pair. Bacterial strain typing was conducted by comparing each sequence with all reference sequences for four bacterial species $(K$. pneumoniae, $K$. aerogenes, E. cloacae, and $E$. coli), and phylogenies of the samples were drawn using the $k$-merbased tree construction method according to the neighbor-joining algorithm. To show consistency between genotyping results and phenotypic characteristics, the MIC for imipenem and meropenem of each bacterium was remeasured using Sensititre (Thermo Fisher Scientific, Massachusetts, USA).

Additionally, multilocus sequence typing (MLST) analyses were conducted for each CP-CRE index patient-secondary patient pair and CP-CRE index patient-CNP-CRE detected patient pair to investigate whether plasmid transfer of carbapenemases had occurred. MLST was performed using the NGS-MLST tool in the CLC Genomics Workbench. Each PubMLST scheme was applied to each species for strain typing. Several housekeeping genes (gapA, infB, mgh, pgi, phoE, rpoB, and tonB for K. pneumoniae; dinB, icdA, pabB, polB, putP, trpA, and trpB for E. coli; and dnaA, fusA, gyrB, leuS, pyrG, rp/B, and $r p o B$ for $E$. cloacae) were selected for MLST, and several antibiotic resistance genes (KPC, OXA, Cefotaximase-Munich (CTX-M), Temoinera (TEM), and Sulfhydryl Variable (SHV)) were used for plasmid analysis. Primers were designed for these housekeeping and resistance genes, and nested PCR assays were conducted for each gene. Amplified sequences of each gene were also analyzed using the method described above.

\section{Data collection and statistical analysis}

Demographic and clinical information about the CP-CRE index and contacted patients was collected from the electronic medical records and infection control department reports of the study hospital. The data were reviewed for the following CP-CRE risk factors: (a) length of hospital stay, (b) previous hospitalizations, (c) previous need for intensive care, $(\mathrm{d})$ presence of indwelling catheters, $(\mathrm{e})$ bed-ridden state, $(\mathrm{f})$ recent invasive procedures, $(\mathrm{g})$ immunocompromised state, and (h) use of antibiotics with broad spectrum coverage [16-20].

All descriptive and statistical analyses were performed using Predictive Analytics Software for Windows (version 25.0; SPSS Inc., Chicago, IL, USA), and p-values less than 0.05 were considered significant. 


\section{Results}

A total of 66 patients had CRE-positive culture studies, carbapenemase NP tests, and carbapenemase PCR tests between January 1, 2017 and December 31, 2019 (Figure 1). Among them, 19 patients either had no contact with other patients, or all contacts had been discharged from the hospital before CP-CRE-positive results came out. The remaining 47 patients with CP-CRE were therefore considered to be index patients, with 152 patients and 54 healthcare workers potentially contacted. Among the 152 contacted patients, 135 (88.8\%) did not have CRE, 10 (6.6\%) had CNP-CRE, and 7 (4.6\%) had CP-CRE. None of the healthcare workers had CRE. Of the 7 CP-CRE-positive patients, four had the same types of carbapenemase as the CP-CRE index patients they were in contact with (all of which were KPC).

\section{Characteristics of CP-CRE index and contacted patients}

The median hospital stay for CP-CRE index patients before CP-CRE-positive results was 6 days (interquartile range, 2-12 days) (Table 1). Stool specimens were used most often for CP-CRE detection (21, 44.7\%), followed by respiratory samples $(9,19.1 \%)$, and urine samples $(6,12.8 \%)$. Among the 47 index patients, Klebsiella pneumoniae was detected in 29 (61.7\%) patients, Escherichia coli in 7 (14.9\%), and Enterobacter species in 7 (14.9\%) patients. KPC was the most common carbapenemase produced $(32,68.1 \%)$, followed by NDM $(15,31.9 \%)$, and OXA-48 (2, 4.3\%). Two patients had both NDM and OXA-48.

The median hospital stay for contacted patients before CP-CRE screening was 12 days (interquartile range 7-23 days), and the median duration the room was shared with a CP-CRE index patient was 3 days (interquartile range 2-7 days) (Table 2). Seventy-eight (51.3\%) patients who were in close contact with an index patient had a history of previous hospitalization, and 27 (17.8\%) had intensive care within the previous 3 months. Fifty-eight (38.2\%) patients used anti-pseudomonal penicillins for more than 3 days within 1 month of CPE surveillance. For 56 (36.8\%), 45 (29.6\%), 35 (23.0\%), and 30 (19.7\%) patients, third or fourth generation cephalosporins, quinolones, glycopeptides, and carbapenems were administered, respectively.

\section{Genomic evaluation in isolates from CP-CRE index and contacted patients}

Only 7 contacted patients had CP-CRE-positive surveillance tests, of whom 4 were secondary patients, and ten had CNP-CRE-positive tests. As described above, WGS with remeasurement of the MIC and MLST were conducted in the 4 CP-CRE index patient-secondary patient pairs with the same carbapenemase types and the 7 CP-CRE index patient-CNP-CRE-detected patient pairs. A total of 22 CP-CRE index patients, secondary patients, and CNP-CREdetected patients with available specimen cultures or CP-CRE surveillance results were subjected to genomic evaluation (Table 3). There were 10 clusters in total, and each cluster was composed of 2 or 3 patients.

From the 22 samples, each bacterium was genetically typed to all reference sequences of four bacterial species ( $K$. pneumoniae, $K$. aerogenes, E. cloacae, and E. coli ), and a phylogenetic tree was constructed based on the bacterial strain typing results by $k$-mer-based tree construction (Figure 2). CP-CRE index patient-CP-CRE-detected patient pairs, such as (I-2, C-2), (I-4, C-4), and (I-7, C-7) showed a genetic distance of less than 0.0005 from each other, and they were all reported to be K. pneumoniae. However, (I-3, C-3), another CP-CRE index patient-secondary patient pair, showed a genetic distance of 0.978 from each other, with I-3 classified as E. coliand C-3 as K. pneumoniae.

In terms of time and place, $(\mathrm{I}-5, \mathrm{I}-10)$ and (I-1, C-9-1) were not related, but the genetic distance between them was less than 0.0005. In addition, (I-6, C-6) and (I-8, C-8) were CP-CRE index patient-CNP-CRE-detected patient pairs, but they were genetically related to each other. In other clusters of CP-CRE index patient-CNP-CRE-detected patients 
pairs, such as (I-1, C-1-1, C-1-2), (I-5, C-5), (I-9, C-9-1, C-9-2), and (I-10, C-10), there was little genetic agreement between the bacteria found in the index patients and the CNP-CRE-detected patients.

To confirm consistency between genotyping results and phenotypic characteristics, the MICs of imipenem and meropenem in each sample were remeasured (Table 4). Using the essential agreement definition of $\pm 1 \log _{2}$ dilution error, (I-2, C-2), (I-4, C-4), and (I-7, C-7) showed similar MIC results between the CP-CRE index patient and the CP-CRE detected patients. The MIC results for other pairs, including (I-3, C-3), however, did not show accordance between the index patient and the detected patient. Although (I-3, C-3) was classified as a CP-CRE index patient-secondary patient pair, I-3 and C-3 were not in accordance with the carbapenem MIC, which could suggest a mismatch between their resistance genes or plasmids.

Results from the MLST, including plasmid analysis, also were similar to the WGS and MIC remeasurement results. The pairs of (I-2, C-2), (I-4, C-4), and (I-7, C-7) between the index and contacted patient were in accordance with the distribution of housekeeping genes and resistance genes in plasmids and the types of carrying plasmids (Supplementary Table 1). Based on the WGS and carbapenem MIC remeasurement results, with the additional MLST, the actual rate of CP-CRE transmission in CP-CRE surveillance was calculated as 1.5\% (3/206) (95\% confidence interval, $0.3 \%-4.2 \%)$.

\section{Clinical characteristics of CPE-transmitted cases}

There were only three CP-CRE transmissions confirmed by WGS and MIC remeasurement among the 206 patients and healthcare workers in contact with CPE-positive patients, so it was impossible to analyze the risk factors for CPCRE transmission. Instead, the clinical characteristics of the three CP-CRE transmission cases are described in detail in a separate table (Supplementary Table 2). The median duration a room was shared with an index patient in the CP-CRE-transmitted patients and non-transmitted patients were 4 days (interquartile range 2-4 days) and 3 days (interquartile range 2-7 days), respectively. There was no significant difference between the two groups using the Mann-Whitney U-test ( $\mathrm{p}$-value $=0.857)$.

\section{Discussion}

The increasing prevalence of CP-CRE is a global concern, resulting in strict guidelines due to the possibility of plasmid transfer to other bacteria [21]. Therefore, CP-CRE transmission and screening guidelines have been implemented across the world. In Korea, when a source patient with CP-CRE is detected, the guidelines recommend isolation in a single room with contact precaution, and all potentially contacted healthcare workers and patients should undergo CP-CRE screening tests [11]. However, the rate of CP-CRE-positive screening tests has not been well studied, though a few studies have reported the rate to be between $2.0 \%$ and $3.2 \%[12,13]$.

In this study, the actual rate of CP-CRE transmission was investigated using WGS with MIC remeasurement. The CPCRE transmission rate was $2.0 \%$ (3/152; $95 \%$ confidence interval, $0.4-5.6 \%)$ in patients who shared a room with an index patient. The 54 healthcare workers tested were all negative for CP-CRE ( $95 \%$ confidence interval, 0.0-0.1\%). The calculated rate of CP-CRE transmission was relatively low, so the benefit of CP-CRE screening may not be high. Considering that the rate of CP-CRE transmission was zero in healthcare workers, it can be inferred that not all CPCRE contacted healthcare workers must undergo CP-CRE screening. Moreover, the three cases of CP-CREtransmission showed similar MIC results between the CP-CRE index patients and CP-CRE-detected patients. This might suggest that genotypic identity implies an accordance with phenotypic traits, such as antibiotic resistance and transmission of carbapenemase between index and contacted patients. 
The transmission rate of CP-CRE could be reduced by the implementation of standard infection control measures, especially strict hand hygiene [22]. Poor hand hygiene among healthcare workers could significantly increase CPCRE transmission [22]; therefore, the compliance rate for hand hygiene among medical staff in hospitals should be reviewed. From 2017 to 2019, the rate of hand hygiene observation among healthcare workers for each year was approximately $95.0 \%$ in the study hospital. Our medical staff had good compliance with hand hygiene, and these rates were maintained for 3 years. Moreover, the three cases of CP-CRE transmission occurred in May, August, and October, 2018. While these cases occurred in similar periods, they were not linked epidemiologically with one another since they did not share the same pathways as each of the CP-CRE index patient-CP-CRE-detected patient pairs. Consequently, they were not considered CP-CRE outbreaks.

WGS analysis showed that CP-CRE from 75\% (3/4) of the secondary patients was in accordance with CP-CRE from the index patients. Therefore, the transmission of plasmids carrying carbapenemase genes between index and secondary patients is possible. In addition, some pairs on the phylogenetic tree were not related to each other in terms of time and place, but they showed genetic identity with each other. This phenomenon could indicate that some bacterial strains may colonize the general environment of the hospital and be transmitted to inpatients.

Several studies have identified risk factors for CP-CRE in a single patient. One matched case-control study investigated 58 patients with CP-CRE among 621,623 admitted patients between 2011 and 2016, and found the following risk factors for CP-CRE: length of hospital stay more than 20 days, history of hospital admission within 1 year, and the use of antibiotics for more than 10 days [16]. Another retrospective study analyzing 303 CP-CRE patients and 5,929 control patients found that the risk factors for CP-CRE were long inpatient stays, mechanical ventilation, dialysis, and exposure to broad-spectrum antibiotics [20]. However, few studies have investigated the risk factors for transmission of CP-CRE in a shared room, and factors precipitating CP-CRE transmission have not been clarified. Risk factors for the transmission of multidrug-resistant bacteria have been reported in several studies, suggesting the duration of stay in the same room and environmental contamination to be likely candidates $[12,23-26]$. One prospective study was conducted on 29 methicillin-resistant Staphylococcus aureus (MRSA) index patients and 84 household contacts between 2005 and 2007, proposing that prolonged exposure to MRSA index patients at home was a significant risk factor for MRSA transmission [23]. In this study, the duration of sharing a room was not significantly different between the CP-CRE-positive and CP-CRE-negative patients. However, other previous studies have revealed that the contaminated environment of a shared room could influence CP-CRE transmission in a hospital $[24,26]$.

This study has several limitations. First, there were only a few CP-CRE-transmission cases, so it was impossible to identify significant risk factors for transmission. Second, environmental cultures in the environment around where CP-CRE index patients stayed was not conducted; therefore, information about environmental contamination could not be investigated. Moreover, several factors that could affect the spread of CP-CRE in a hospital, such as the number of antibiotic stewardship cases per 1000 patient-days, the ratio of caregivers to patients, and the amount of hydroalcoholic products used were not investigated [22]. Further studies considering environmental cultures, the enforcement rate of antibiotic stewardship, the ratio of caregivers to patients, and a greater number of CP-CRE transmitted cases should be conducted.

\section{Conclusions}

The rate of transmission of CP-CRE to patients who shared a room with CP-CRE-positive patients was calculated as $2.0 \%$ based on genetic and phenotypic analyses in a tertiary teaching hospital, while the rate of transmission to

Page $7 / 19$ 
contacted healthcare workers was zero. Considering the low transmission rate of CP-CRE in shared rooms, screening tests for patients contacted to CP-CRE could be implemented in specific cases only.

\section{Abbreviations}

CRE: Carbapenem-resistant Enterobacteriaceae; CP-CRE: Carbapenemase-producing carbapenem-resistant Enterobacteriaceae; CNP-CRE: Carbapenemase-non-producing carbapenem-resistant Enterobacteriaceae; PCR: Polymerase chain reaction; MIC: Minimal inhibitory concentration; KPC: Klebsiella pneumoniae carbapenemase; IMP: Imipenemase; VIM: Verona integron-encoded metallo- $\beta$-lactamase; NDM: New Delhi metallo- $\beta$-lactamase; OXA: Oxacillinase; WGS: Whole genome sequencing; MLST: Multilocus sequence typing; MRSA: Methicillin-resistant Staphylococcus aureus

\section{Declarations}

\section{Acknowledgements}

Not applicable.

\section{Authors' contributions}

EC and NJK designed the study, collected the data and wrote and revised the manuscript. HEC, ISS and KUP analyzed and interpreted the data and revised the manuscript. YRO and EHC collected the data. CKK, PGC, WBP and MO interpreted the data and revised the manuscript.

\section{Author's information}

Not applicable.

\section{Funding}

This work was supported by grants from the research funds of Seoul National University Hospital (Grant No. 052020-0010) and the Korea Disease Control and Prevention Agency (Grant No. 2019-ER5402-01 \& Grant No. 2020ER5403-00).

\section{Availability of data and materials}

All data generated or analyzed during this study are included in this published article.

\section{Ethics approval and consent to participate}

This study was approved by the institutional review board of the hospital (IRB No. H-2004-135-1117). The informed consent requirement was waived, since this study was retrospective, involved no interaction with patients, and was considered to be of minimal risk.

\section{Consent for publication}

Not applicable.

\section{Competing interests}


The authors declare that they have no competing interests.

\section{Author details}

${ }^{1}$ Department of Internal Medicine, Seoul National University College of Medicine, Seoul, Republic of Korea. ${ }^{2} \mathrm{PHiCS}$ Institute, Seoul, Republic of Korea. ${ }^{3}$ Center for Infection Control and Prevention, Seoul National University Hospital, Seoul, Republic of Korea. ${ }^{4}$ Department of Pediatrics, Seoul National University College of Medicine, Seoul, Republic of Korea. ${ }^{5}$ Department of Laboratory Medicine, Seoul National University College of Medicine, Seoul, Republic of Korea.

\section{References}

1 Papp-Wallace KM, Endimiani A, Taracila MA, et al. Carbapenems: Past, present, and future. Antimicrob Agents Chemother 2011;55:4943-60. doi:10.1128/AAC.00296-11

2 Hawkey PM, Livermore DM. Carbapenem antibiotics for serious infections. BMJ 2012;344:1-7. doi:10.1136/bmj.e3236

3 Li Y, Sun Q-L, Shen Y, et al. Rapid Increase in Prevalence of Carbapenem-Resistant Enterobacteriaceae (CRE) and Emergence of Colistin Resistance Gene mcr-1 in CRE in a Hospital in Henan, China. J Clin Microbiol 2018;56:e01932-17. doi:10.1128/JCM.01932-17

4 Thaden JT, Lewis SS, Hazen KC, et al. Rising Rates of Carbapenem-Resistant Enterobacteriaceae in Community Hospitals: A Mixed-Methods Review of Epidemiology and Microbiology Practices in a Network of Community Hospitals in the Southeastern United States. Infect Control Hosp Epidemiol 2014;35:978-83. doi:10.1086/677157

5 Wan PJ, Jae LS, Hyungmin L. Status of carbapenemase-producing enterobacteriaceae incidences in Korea, 20152016. Public Heal Wkly Report, Korea Dis Control Prev Agency 2017;10:1243-7.

6 Martin A, Fahrbach K, Zhao Q, et al. Association between carbapenem resistance and mortality among adult, hospitalized patients with serious infections due to enterobacteriaceae: Results of a systematic literature review and meta-analysis. Open Forum Infect Dis 2018;5:1-9. doi:10.1093/ofid/ofy150

7 Matthew E. Falagas, Giannoula S. Tansarli, Drosos E. Karageorgopoulos, et al. Deaths Attributable to Carbapenem-Resistant Enterobacteriaceae Infections. Emerg Infect Dis 2014;20:11705.https://www.ncbi.nlm.nih.gov/pmc/articles/PMC4073868/pdf/12-1004.pdf

8 Queenan AM, Bush K. Carbapenemases: The versatile $\beta$-lactamases. Clin Microbiol Rev 2007;20:440-58. doi:10.1128/CMR.00001-07

9 Pitout JDD. Multiresistant Enterobacteriaceae: new threat of an old problem. Expert Rev Anti Infect Ther 2008;6:657-69. doi:10.1586/14787210.6.5.657

10 Nordmann P. Carbapenemase-producing Enterobacteriaceae: overview of a major public health challenge. Med Mal Infect 2014;44:51-6. doi:10.1016/j.medmal.2013.11.007

11 Korea Disease Control and Prevention Agency. Guidelines for control of patients with Carbapenemase producing Carbapenem-Resistant Enterobacteriaceae. 2020. 
12 Jamal AJ, Faheem A, Farooqi L, et al. Household Transmission of Carbapenemase-Producing Enterobacterales (CPE) in Ontario, Canada. Clin Infect Dis Published Online First: 1 September 2020. doi:10.1093/cid/ciaa1295

13 Park JW, Kwak SH, Jung J, et al. The rate of acquisition of carbapenemase-producing Enterobacteriaceae among close contact patients depending on carbapenemase enzymes. Infect Chemother 2020;52:39-47. doi:10.3947/ic.2020.52.1.39

14 Wayne PA. Clinical and Laboratory Standards Institute Methods for Dilution Antimicrobial Susceptibility Tests for Bacteria That Grow Aerobically Standard,, Approval CDM-A.; Clin Lab Stand Inst 2018;:91.

15 EUCAST. Testing Breakpoint tables for interpretation of MICs and zone diameters. 2020. https://www.eucast.org/fileadmin/src/media/PDFs/EUCAST_files/Breakpoint_tables/v_10.0_Breakpoint_Tables.pdf

16 Lusignani LS, Presterl E, Zatorska B, et al. Infection control and risk factors for acquisition of carbapenemaseproducing enterobacteriaceae. A 5 year ( 2011 - 2016 ) case-control study. Antimicrob Resist Infect Control 2020;9:1-10.

17 Falagas ME, Rafailidis PI, Kofteridis D, et al. Risk factors of carbapenem-resistant Klebsiella pneumoniae infections: a matched case - control study. 2007;:1124-30. doi:10.1093/jac/dkm356

18 Mariappan S, Sekar U, Kamalanathan A. Carbapenemase-producing Enterobacteriaceae: Risk factors for infection and impact of resistance on outcomes. Int J Appl basic Med Res 2017;7:32-9. doi:10.4103/2229516X.198520

19 Nicolas-Chanoine M-H, Vigan M, Laouénan C, et al. Risk factors for carbapenem-resistant Enterobacteriaceae infections: a French case-control-control study. Eur J Clin Microbiol Infect Dis 2019;38:383-93. doi:10.1007/s10096-018-3438-9

20 Mathers AJ, Vegesana K, German-Mesner I, et al. Risk factors for Klebsiella pneumoniae carbapenemase (KPC) gene acquisition and clinical outcomes across multiple bacterial species. J Hosp Infect 2020;104:456-68. doi:10.1016/j.jhin.2020.01.005

21 Mataseje LF, Abdesselam K, Vachon J, et al. Results from the Canadian Nosocomial infection surveillance program on Carbapenemase-producing Enterobacteriaceae, 2010 to 2014. Antimicrob Agents Chemother 2016;60:6787-94. doi:10.1128/AAC.01359-16

22 Legeay C, Thépot-Seegers V, Pailhoriès H, et al. Is cohorting the only solution to control carbapenemaseproducing Enterobacteriaceae outbreaks? A single-centre experience. J Hosp Infect 2018;99:390-5. doi:10.1016/j.jhin.2018.02.003

23 Mollema FPN, Richardus JH, Behrendt M, et al. Transmission of methicillin-resistant Staphylococcus aureus to household contacts. J Clin Microbiol 2010;48:202-7. doi:10.1128/JCM.01499-09

24 Clarivet B, Grau D, Jumas-Bilak E, et al. Persisting transmission of carbapenemase-producing Klebsiella pneumoniae due to an environmental reservoir in a university hospital, France, 2012 to 2014. Eurosurveillance 2016;21. doi:10.2807/1560-7917.ES.2016.21.17.30213 
25 Smismans A, Ho E, Daniels D, et al. New environmental reservoir of CPE in hospitals. Lancet Infect Dis 2019;19:580-1. doi:10.1016/S1473-3099(19)30230-0

26 De Geyter D, Blommaert L, Verbraeken N, et al. The sink as a potential source of transmission of carbapenemaseproducing Enterobacteriaceae in the intensive care unit. Antimicrob Resist Infect Control 2017;6:1-6. doi:10.1186/s13756-017-0182-3

\section{Tables}

Table 1 Demographic and clinical characteristics of CP-CRE index patients 
Characteristics

Age (median) (years, IQR)

Sex

Male

Female

Admitted department

Emergency medicine

Internal medicine

General surgery

Neurology

Others

Duration of hospitalization till CP-CRE was detected (median) (days, IQR)

Underlying diseases

Hematologic diseases or solid malignancy

Chronic kidney diseases

Chronic heart diseases

Chronic liver diseases

Chronic pulmonary diseases

Diabetes mellitus

Stroke

Organ transplantation

Bacterial species

Klebsiella pneumoniae

Escherichia coli

Enterobacter species

Others

Types of carbapenemases

KPC

NDM

OXA-48
CP-CRE index patients $(\mathrm{N}=47)(\%)$

$60.6(52.5-72.5)$

22 (46.8)

25 (53.2)

15 (31.9)

9 (19.1)

$9(19.1)$

7 (14.9)

7 (14.9)

$6(2-12)$

20 (42.6)

10 (21.3)

9 (19.1)

5 (10.6)

3 (6.4)

6 (12.8)

6 (12.8)

4 (8.5)

29 (61.7)

7 (14.9)

7 (14.9)

4 (8.5)

32 (68.1)

15 (31.9)

2 (4.3)

$\mathrm{N}$, number; IQR, interquartile range; CP-CRE, carbapenemase-producing carbapenem-resistant Enterobacteriaceae; KPC, Klebsiella pneumoniae carbapenemase; NDM, New Delhi metallo-beta-lactamase; OXA, oxacillinase 
Table 2 Demographic and clinical characteristics of patients contacted to CP-CRE through a shared room 
Characteristics

Age (median) (years, IQR)

Sex

Male

Female

Contacted department

Emergency medicine

Internal medicine

General surgery

Neurology

Orthopedics

Neurosurgery

Others

Duration of hospitalization until CP-CRE surveillance (median) (days, IQR)

Duration of sharing a room with CP-CRE index patients (median) (days, IQR)

Underlying diseases

Hematologic diseases or solid malignancy

Chronic kidney diseases

Chronic heart diseases

Chronic liver diseases

Chronic pulmonary diseases

Diabetes mellitus

Stroke

Organ transplantation

Previous hospitalization within 3 months

Previous intensive care within 3 months

Invasive procedure during the hospital course ${ }^{a}$

Use of broad spectrum antibiotics ${ }^{b}$

Anti-pseudomonal penicillins

$3^{\text {rd }}$ or $4^{\text {th }}$ generation cephalosporins
$58(38.2)$

CP-CRE contact patients $(\mathrm{N}=152)$

$(\%)$

$63.0(49.5-72.0)$

$104(68.4)$

$48(31.6)$

$53(34.9)$

$28(18.4)$

$25(16.4)$

$18(11.8)$

$12(7.9)$

$9(5.9)$

7 (4.6)

12 (7-23)

$3(2-7)$

66 (43.4)

$26(17.1)$

20 (13.2)

27 (17.8)

16 (10.5)

43 (28.3)

21 (13.8)

10 (6.6)

78 (51.3)

27 (17.8)

103 (67.8)

$56(36.8)$ 
Colistin

$5(3.3)$

Tigecycline

Glycopeptides $35(23.0)$

$\mathrm{N}$, number; IQR, interquartile range

a Any type of percutaneous drainage and transfemoral intervention, central line insertion, intracardiac device implantation, intubation or tracheostomy, endoscopic evaluation, biopsy or centesis, and surgery were included.

${ }^{b}$ Used for more than 3 days within the previous month

Table 3 Clusters of CP-CRE index patients, secondary patients, and CNP-CRE-detected patients who underwent WGS analysis 


\begin{tabular}{|c|c|c|c|c|c|c|}
\hline $\begin{array}{l}\text { Number of } \\
\text { cluster }\end{array}$ & $\begin{array}{l}\text { Number of } \\
\text { colony }\end{array}$ & Sex & Age & Specimen & Bacterial species & $\begin{array}{l}\text { Types of } \\
\text { carbapenemases }\end{array}$ \\
\hline \multirow[t]{3}{*}{$2017-6$} & $\mathrm{I}-1$ & $\mathrm{~F}$ & 71 & Sputum & $\begin{array}{l}\text { Klebsiella } \\
\text { pneumoniae }\end{array}$ & NDM-5, OXA-181 \\
\hline & $C-1-1$ & $\mathrm{~F}$ & 65 & Rectal & $\begin{array}{l}\text { Klebsiella } \\
\text { aerogenes }\end{array}$ & negative \\
\hline & $\mathrm{C}-1-2$ & $\mathrm{~F}$ & 72 & Rectal & $\begin{array}{l}\text { Klebsiella } \\
\text { aerogenes }\end{array}$ & negative \\
\hline \multirow[t]{2}{*}{$2018-3$} & $\mathrm{I}-2$ & M & 72 & Rectal & $\begin{array}{l}\text { Klebsiella } \\
\text { pneumoniae }\end{array}$ & $\mathrm{KPC}$ \\
\hline & C-2 & $\mathrm{F}$ & 78 & Rectal & $\begin{array}{l}\text { Klebsiella } \\
\text { pneumoniae }\end{array}$ & KPC \\
\hline \multirow[t]{2}{*}{$2018-5$} & $\mathrm{I}-3$ & $\mathrm{~F}$ & 78 & Urine & Escherichia coli & $\mathrm{KPC}$ \\
\hline & C-3 & $\mathrm{F}$ & 55 & Rectal & $\begin{array}{l}\text { Klebsiella } \\
\text { pneumoniae }\end{array}$ & KPC \\
\hline \multirow[t]{2}{*}{$2018-8$} & $\mathrm{I}-4$ & M & 66 & TTA & $\begin{array}{l}\text { Klebsiella } \\
\text { pneumoniae }\end{array}$ & $\mathrm{KPC}$ \\
\hline & C-4 & M & 81 & Rectal & $\begin{array}{l}\text { Klebsiella } \\
\text { pneumoniae }\end{array}$ & $\mathrm{KPC}$ \\
\hline \multirow[t]{2}{*}{ 2018-11 } & $1-5$ & M & 59 & Sputum & $\begin{array}{l}\text { Klebsiella } \\
\text { pneumoniae }\end{array}$ & NDM \\
\hline & C-5 & M & 23 & Rectal & $\begin{array}{l}\text { Klebsiella } \\
\text { pneumoniae }\end{array}$ & Negative \\
\hline \multirow[t]{2}{*}{ 2018-13 } & $1-6$ & M & 50 & TTA & $\begin{array}{l}\text { Enterobacter } \\
\text { cloacae }\end{array}$ & NDM \\
\hline & C-6 & M & 70 & Rectal & $\begin{array}{l}\text { Enterobacter } \\
\text { cloacae }\end{array}$ & Negative \\
\hline \multirow[t]{2}{*}{ 2018-14 } & $1-7$ & M & 70 & sputum & $\begin{array}{l}\text { Klebsiella } \\
\text { pneumoniae }\end{array}$ & KPC \\
\hline & C-7 & M & 38 & rectal & $\begin{array}{l}\text { Klebsiella } \\
\text { pneumoniae }\end{array}$ & KPC \\
\hline \multirow[t]{2}{*}{ 2018-19 } & $\mathrm{I}-8$ & M & 23 & urine & $\begin{array}{l}\text { Klebsiella } \\
\text { pneumoniae }\end{array}$ & $\mathrm{KPC}$ \\
\hline & $\mathrm{C}-8$ & M & 23 & rectal & $\begin{array}{l}\text { Klebsiella } \\
\text { pneumoniae }\end{array}$ & negative \\
\hline \multirow[t]{3}{*}{$2018-20$} & $1-9$ & M & 56 & BAL & $\begin{array}{l}\text { Klebsiella } \\
\text { pneumoniae }\end{array}$ & KPC \\
\hline & C-9-1 & M & 71 & Rectal & $\begin{array}{l}\text { Klebsiella } \\
\text { pneumoniae }\end{array}$ & negative \\
\hline & C-9-2 & $M$ & 94 & Rectal & $\begin{array}{l}\text { Klebsiella } \\
\text { pneumoniae }\end{array}$ & negative \\
\hline 2019-3 & $\mathrm{I}-10$ & $M$ & 83 & Rectal & $\begin{array}{l}\text { Klebsiella } \\
\text { pneumoniae }\end{array}$ & KPC \\
\hline
\end{tabular}




C-10 M 63 Rectal $\begin{aligned} & \text { Klebsiella } \\ & \text { pneumoniae }\end{aligned}$

I, index; C, colony; F, female; M, male; KPC, Klebsiella pneumoniae carbapenemase; NDM, New Delhi metallo-betalactamase; OXA, oxacillinase; TTA, transtracheal aspiration; BAL, bronchoalveolar lavage

Table 4 MIC remeasurement results for imipenem and meropenem

\begin{tabular}{|c|c|c|c|}
\hline $\begin{array}{l}\text { Number of } \\
\text { Colony }\end{array}$ & Bacterial species & Imipenem MIC ( $\nabla / m I)$ & 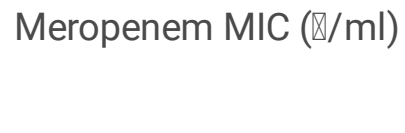 \\
\hline I-1 & K. pneumoniae & $32(\mathrm{R})$ & $128(\mathrm{R})$ \\
\hline C-1-1 & K. aerogenes & $4(\mathrm{R})$ & $1(\mathrm{~S})$ \\
\hline C-1-2 & K. aerogenes & $4(\mathrm{R})$ & $2(I)$ \\
\hline $1-2$ & K. pneumoniae & $8(R)$ & $16(\mathrm{R})$ \\
\hline $\mathrm{C}-2$ & K. pneumoniae & $16(\mathrm{R})$ & $8(\mathrm{R})$ \\
\hline $1-3$ & E. coli & $2(I)$ & $2(I)$ \\
\hline C-3 & K. pneumoniae & $16(\mathrm{R})$ & $32(R)$ \\
\hline $1-4$ & K. Pneumoniae & $4(\mathrm{R})$ & $2(I)$ \\
\hline C-4 & K. Pneumoniae & $4(\mathrm{R})$ & $2(I)$ \\
\hline $\mid-5$ & K. Pneumoniae & $64(\mathrm{R})$ & $128(\mathrm{R})$ \\
\hline C-5 & K. Pneumoniae & $\leq 0.5(\mathrm{~S})$ & $2(I)$ \\
\hline $1-6$ & E. cloacae & $4(\mathrm{R})$ & $2(I)$ \\
\hline C-6 & E. cloacae & $\leq 0.5(\mathrm{~S})$ & $\leq 0.5(\mathrm{~S})$ \\
\hline $1-7$ & K. pneumoniae & $8(R)$ & $8(\mathrm{R})$ \\
\hline $\mathrm{C}-7$ & K. pneumoniae & $8(R)$ & $8(\mathrm{R})$ \\
\hline $1-8$ & K. pneumoniae & $32(R)$ & $64(\mathrm{R})$ \\
\hline C-8 & K. pneumoniae & $2(I)$ & $16(R)$ \\
\hline $1-9$ & K. pneumoniae & $8(R)$ & $4(\mathrm{R})$ \\
\hline C-9-1 & K. pneumoniae & $4(\mathrm{R})$ & $8(\mathrm{R})$ \\
\hline C-9-2 & K. pneumoniae & $\leq 0.5(\mathrm{~S})$ & $4(\mathrm{R})$ \\
\hline $\mathrm{I}-10$ & K. pneumoniae & $128(\mathrm{R})$ & $\geq 256(\mathrm{R})$ \\
\hline C-10 & K. pneumoniae & $4(\mathrm{R})$ & $4(\mathrm{R})$ \\
\hline
\end{tabular}

I, index; C, colony; R, resistant; I, intermediate; S, susceptible

\section{Figures}




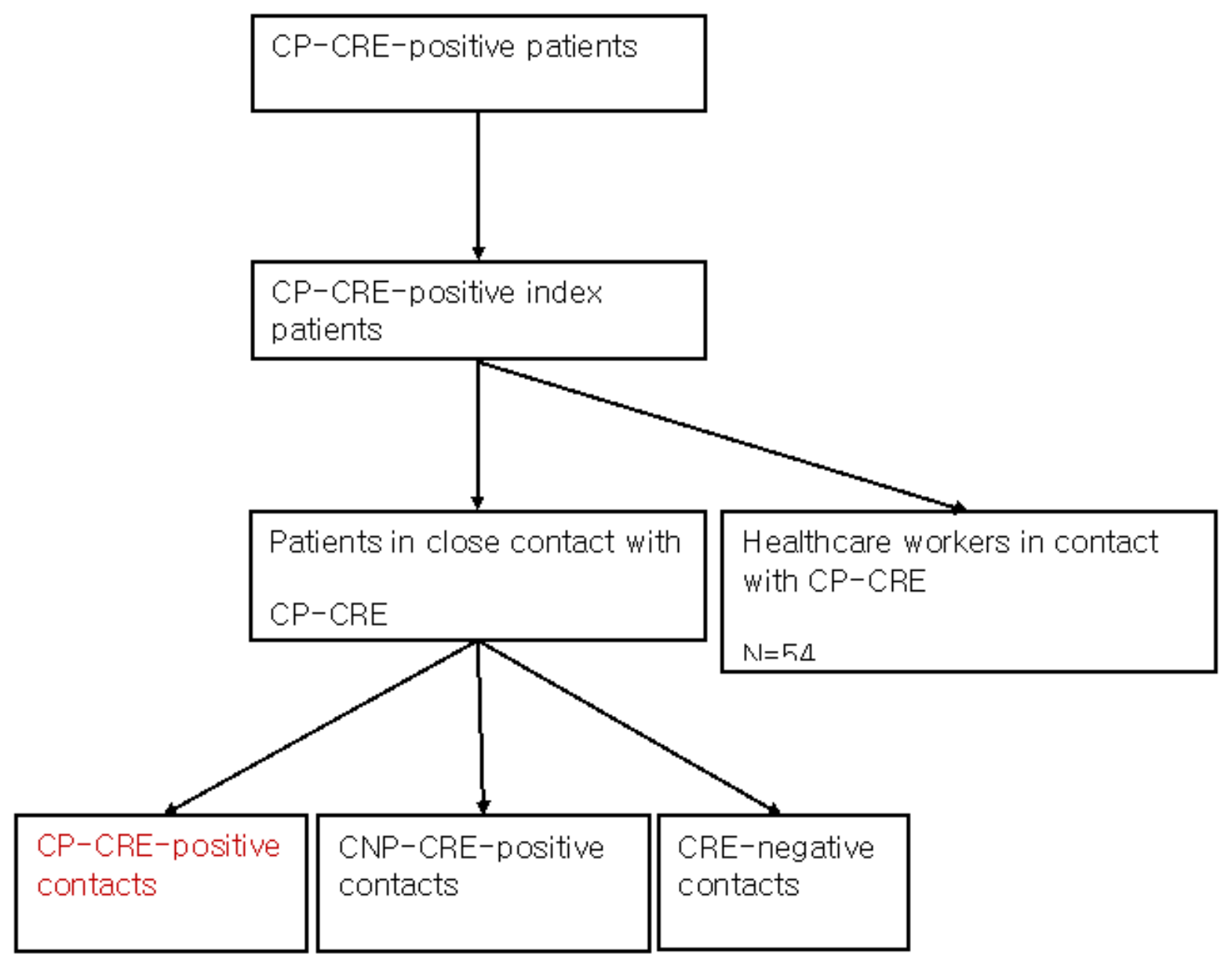

Figure 1

Flow diagram of screening. N, number 


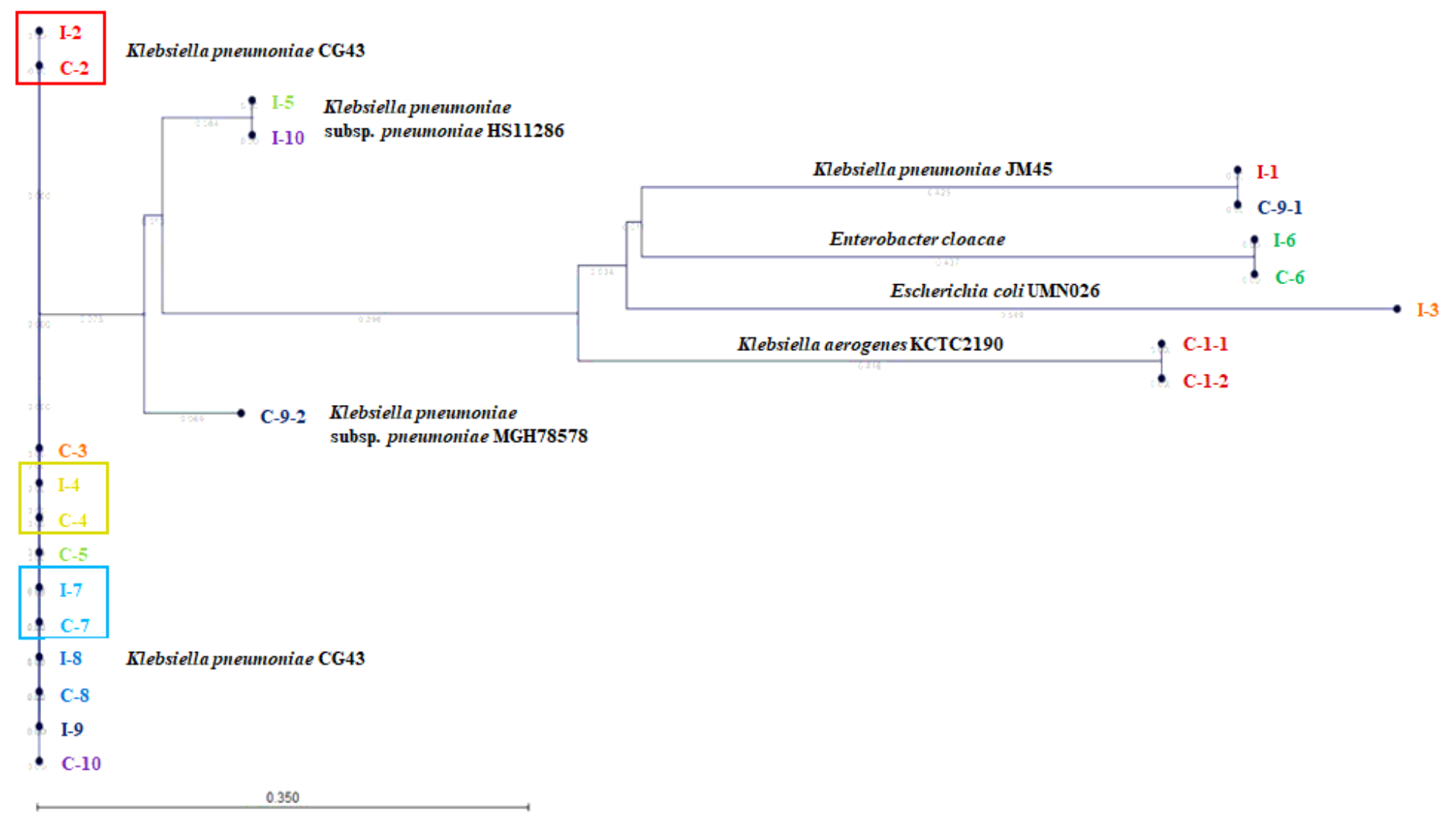

\section{Figure 2}

The phylogenetic tree of CP-CRE index patients and those in close contact who were CRE-positive. I, index; C, colony The phylogenetic tree of CP-CRE index patients and those in close contact who were CRE-positive, which was drawn using the k-mer-based tree construction method. The numbers below the horizontal lines indicate the genetic distance between the two bacterial colonies represented by dots. The strain typing results based on WGS are shown with the bacterial names. The colonies in the colored boxes represent the pairs considered to be the transmitted cases.

\section{Supplementary Files}

This is a list of supplementary files associated with this preprint. Click to download.

- CPETransmissionSupplementaryTablesARIC210424.docx 